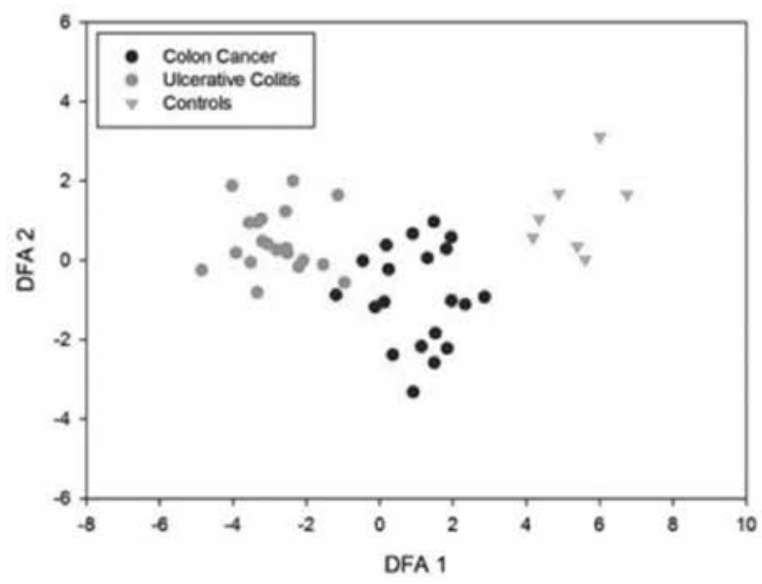

Abstract PTH-018 Figure 1

Conclusion This pilot study suggests that both E-nose and FAIMS offer a different and non-invasive approach with high potential to identify those with CRC.

Disclosure of Interest None Declared.

\section{PTH-019 COLONIC STENTING IN A DGH WITHOUT A RADIOLOGIST SUPPORT}

doi:10.1136/gutjnl-2013-304907.507

1."R K Mehmood, 'P Kirkbride, 'E Jehangir. 'Colorectal Surgery, West Cumberland Hospital, Whitehaven, UK

Introduction The aim of this study is to assess our outcomes of colonic stenting for colorectal malignancies in a DGH where we provide stenting without the support of interventional radiologist. NICE guidelines recommend facilities and services should be established to provide stenting for patients with intestinal obstruction, particularly those with serious co-morbidity to avoid emergency surgery ${ }^{1}$. We run a colorectal laparoscopic unit adequately according to NICE recommendations although we do not have support of interventional radiologist

Methods It is retrospective study of all stents which were inserted in a DGH by a surgeon between 2004 and 2012. All patients had presented with acute bowel obstruction caused by malignant colorectal diseases and structuring. Data was collected prospectively by the colorectal specialist nurse on a pre-designed database format.

In the stent insertion no support of interventional radiologist was available. However all stents were inserted by a surgeon with the help of endoscopes and fluoroscopy in X-ray department.

Results Out of 54 patients 51 had successful stenting initially. However on three occasions stenting was not successful. Out of 51 who had successful stenting in three of them stents were not found adequately relieving obstruction so they ended up having stoma. The complete success rate in 48 patients was $88 \%$. The indications of stenting were for relief of acute bowel obstruction, palliation and as a bridge to surgery. Complications were noted in

Seven cases (14.58) including one perforations and one migration, one obstructive symptoms persisted and four had growth of the tumour at the lower end of the stents where restenting was not successful.. There were no incidents of acute or delayed haemorrhage in any patients.

Conclusion This study demonstrated that stenting for obstructing colorectal cancers is a safe and effective method of relieving acute and impending bowel obstruction. It is also a preferred method of converting an acute surgery into an elective surgery by "bridging to surgery" in order to optimise patient's condition before surgery.

Our study also showed that stenting can be provided safely and effectively in a district general hospital by a trained surgeon without the help of interventional radiologist.
Disclosure of Interest None Declared.

\section{REFERENCE}

1. http://www.nice.org.uk/nicemedia/pdf/CSGCCfullguidance.pdf

\section{PTH-020 FACTORS INFLUENCING ABNORMAL GLAND MORPHOGENESIS IN COLORECTAL CANCER (CRC) - TRANSLATIONAL STUDIES}

doi:10.1136/gutjnl-2013-304907.508

${ }^{1, *} \mathrm{R}$ Topley, ${ }^{2} \mathrm{~A}$ Fatehullah, ${ }^{3} \mathrm{R}$ Deevi, ${ }^{4} \mathrm{I}$ Jagan, ${ }^{1} \mathrm{~J}$ Eves, ${ }^{5} \mathrm{M}$ Loughrey, ${ }^{1} \mathrm{~K}$ Arthur, ${ }^{5} \mathrm{M}$ Stevenson, ${ }^{1} \mathrm{~F}$ C Campbell on behalf of Campbell Cancer Morphogensis Group. ${ }^{1}$ CCRCB, Queen's University Belfast, Belfast; ${ }^{2}$ College of Life Sciences, University of Dundee, Dundee, UK; ${ }^{3}$ Cell Biology Lab, University College Cork, Cork, Ireland; ${ }^{4} \mathrm{UHN}$ Toronto, Toronto, Canada; ${ }^{5}$ Queen's University Belfast, Belfast, UK

Introduction Disruption of colorectal gland formation characterises high grade, aggressive CRCs but causal mechanisms remain unclear. Glandular morphogenesis can be modelled in three-dimensional (3D) culture systems that enable investigation of specific oncogenic signals. We have shown that the tumour suppressor PTEN regulates 3D glandular morphogenesis in a Caco- 2 colorectal organotypic model system through effects on the Rho-GTPase cdc42. Cdc42 is activated by specific guanine nucleotide exchange factors (GEFs) and influences gland lumen formation by regulation of apical membrane (AM) assembly. PTENknockdown inhibits cdc42, disrupts AM integrity and induces a vacuolar, multilumen glandular phenotype evocative of high grade CRC. While PTENhas catalytically -active or -inactive functional domains relevant to phosphatidylinositol 3-kinase (PI3K) activity, Caco-2 gland development was unaffected by PI3K signalling.

Methods We used wild type PTEN-expressing Caco-2 cells and isogenic stable PTENknockdown Caco-2 (KD) clones in two- (2D) and three-dimensional (3D) cultures as model systems. Cell membrane localization of specific cdc 42 GEFs was investigated by cell fractionation and immunoblot. Effects of catalytically -active or -inactive PTENmutants on cdc42 activity and/or AM integrity during $3 \mathrm{D}$ morphogenesis were investigated by transfection and confocal microscopy. Apical membrane integrity was assessed in human CRCby semiquantitative score of the AM marker, NHERF1. CRCgland morphology was assessed by a validated grading system

Results PTENexpression enhanced cell membrane recruitment of cdc42 GEFs with a specific role in 3D morphogenesis (Tuba, ITSN2). PTENmutants containing an intact catalytically-inactive C2 domain enhanced cdc42 activity, restored AM integrity and rescued defective morphogenesis of 3D PTEN-KD Caco-2 cultures. Conversely, a C2 domain construct mutated at its CBR3 lipid-binding motif was ineffective. Fundamental attributes of the model system viz, associations between AM integrity and gland morphology were conserved and had prognostic significance in human CRC.

Conclusion PTENdeficiency impairs GEF membrane recruitment, cdc42 activation, apical membrane assembly and CRCglandular morphogenesis in a predictive colorectal cancer model system.

PTEN-cdc42 regulatory pathways influence AM integrity and colorectal glandular morphogenesis. Dissection of these networks may identify molecular targets for novel therapy, aimed at high grade CRC.

Disclosure of Interest None Declared.

\section{PTH-021 MAGNETIC ANAL SPHINCTER - A NOVEL SURGICAL OPTION FOR MANAGEMENT OF FAECAL INCONTINENCE}

doi:10.1136/gutjnl-2013-304907.509

1.2.* R Tevlin, 'A Hanly, ' $\mathrm{J}$ Larkin, ${ }^{1,3} \mathrm{R}$ O'Connell. 'Colorectal Surgery, St. Vincent's University Hospital; ${ }^{2}$ Royal College of Surgeons in Ireland; ${ }^{3}$ Department of Surgery and Surgical Specialities, University College Dublin, Dublin, Ireland 